\title{
Frequency of GSTT1*0 genotype as a non-conjugator phenotype in a group of healthy Iranian people
}

\author{
Monireh Aghajany-Nasab ${ }^{1}$, Siamak Mirab Samiee ${ }^{2,4}$, Mojtaba Panjehpour ${ }^{1,5}$, Aliakbar Malekirad ${ }^{3}$, \\ Ahmad Movahedian-Attar ${ }^{1}$ \\ ${ }^{1}$ Department of Clinical Biochemistry, Isfahan Pharmaceutical Sciences Research Center, School of Pharmacy and Pharmaceutical \\ Sciences, Isfahan University of Medical Sciences, Isfahan, Iran; \\ ${ }^{2}$ Food and Drug Laboratory Research Center, Ministry of Health and Medical Education, Tehran, Iran; \\ ${ }^{3}$ Payame Noor University (P.N.U), Tehran, Iran; \\ ${ }^{4}$ Day General Hospital Laboratory, Tehran, Iran; \\ ${ }^{5}$ Bioinformatics Research Center, Isfahan University of Medical Sciences, Isfahan, Iran. \\ Email: movahedian@pharm.mui.ac.ir
}

Received 17 December 2010; revised 25 January 2011; accepted 29 January 2011.

\section{ABSTRACT}

Glutathione S-transferases (GSTs) are dimeric mainly cytosolic enzymes involved in detoxification of many exogenous and endogenous disease-causing electrophilic substrates. GSTs also have critical role in phase II biotransformation of a number of drugs, xenobiotics and industrial chemicals and protect cellular macromolecules. The evidences supported that human GSTT1 contributes in the deactivation of reactive oxygen species which more likely to be effective in inflammatory diseases, ageing and some non-cancer diseases also different types of cancers. The GSTT1 is genetically deleted in a high percentage of the different ethnic groups. Although this gene is highly conserved during evolution, it is indeed surprising that the GSTT1 deleted gene could be found in high incidence of human population. Conjugator and non-conjugator phenotypes are coincident with this deletion (GSTT1*1 and GSTT1*0 genotype).The consequences of this deletion could be involved in some diseases outcome, toxicology and drug resistances. In this study the Real-time PCR assay and a set of hybridization probes was used as a one-step and accurate method to estimate the frequency of GSTT1*0 genotype as a non-functional phenotype in 90 healthy individuals from the province of Isfahan in Iran. GSTT1 genotypes were identified in DNA samples using fluorogenic Real-Time PCR (LightCycler) followed by online melting curve analysis. The incidence of GSTT1*1(wild type) and GSTT1*0(Null type) were $\mathbf{7 4 . 5} \%$ and $\mathbf{2 5 . 5 \%}$ respectively. No differences in genotypes frequencies were perceived in samples stratified by age and gender $P>0.05$. The results were compared with other ethnic groups to get more insight into the frequency differences of defected carcinogens metabolizer gene due to deletion polymorphism of GSTT1. It has been indicated the incidence of GSTT1*0 in this group of Iran showed significant differences with East Asian and some European and American countries $P<0.05$. The prevalence of GSTT1 null genotype in the study group from Isfahan province of Iran was slightly higher in comparison with other Iranian ethnic group (Iranian Georgian $15.7 \%$ ), but this difference was not significant $\left(\chi^{2}=1.66, P=0.197\right)$. Further experimental investigations are needed to inquiry the clinical implications of GSTT1 genetic polymorphism with consider to significant variability among different ethnicities.

Keywords: GSTT1 Genotyping, Genetic Polymorphism, Real-Time PCR, Ethnic Differences, Iran

\section{INTRODUCTION}

Glutathione S-transferase T1 detoxifies the genotoxic compounds containing an electrophilic center and protects from DNA damage. GSTT protects cells by catalyzing the conjugation of toxic and carcinogenic electrophilic molecules with glutathione that are excreted through kidney [1]. It is hypothesized that reduced GSTs activity is associated with higher incidence of cancer that seems a results of decreased elimination of electrophilic carcinogens [2] Inter-individual differences in the capacity of detoxifying toxins, cancer susceptibility, and drug resistance maybe mediated in part through polymorphic variability in the bioactivation and detoxification of occupational carcinogens, environmental pollutants [3]. Several studies have found GSTs consistency in cancer susceptibility genes [4-6] and few studies report the rela- 
tion of toxicity of chemotherapeutic agents with GSTs inactive genotype [7]. Other studies have shown that GST theta null genotypes individuals are more susceptible to the mutagenic effects of some GSTT1 substrates [8].

The human theta subfamily of GSTs consists of two different genes, GSTT1 and GSTT2, both of them located on chromosome 22q11.2, consisting of 5 exons. GSTT1 has a functional allele GSTT1*1 and a null allele, GSTT1*0. GSTT1*0 is formed by homologous recombination which result in $54 \mathrm{~kb}$ deletion comprising the complete GSTT1 gene so it has an impaired ability to metabolically eliminate carcinogenic compounds. It has been reported that GSTT1*1 presents conjugator phenotype while GSTT1*0 has non-conjugator phenotypes [9]. The gene and associated proteins were found in a definite percentage of the human population with different ethnicities.

The GSTT1*0 genotype has been found to elevate baseline level of sister chromatid exchange (SCE) frequently after exposure to 1, 3-butadiene and haloalkanes. It has been found SCE, in turn, has strong positive correlation with cancer risk [10].

Polymorphism interaction analysis (PIA) indicated that GSTT1 is a polymorphism predicting cancer [9]. Among known substrates metabolized by GST theta, dichloromethane (DCM) is one of the most thoroughly studied [11]. Erythrocytes from some subjects ("conjugators") catalyze the conjugation of DCM with GSH, whereas the remaining individuals ("non-conjugators") do not. Formaldehyde obtained from metabolism of DCM covalently binds to cellular macromolecules like single-stranded DNA, serum albumin, or the N-terminal valine of hemoglobin and form molecular adducts [12]. Formaldehyde-adducted RNAs were found in DCMexposed isolated hepatocytes GSTT1*1 (positive) human but not in hepatocytes from GSTT1*0(negative) donors [13].

1,3-Butadiene, ethylene, drinking water after chlorination, and metabolites of aflatoxin are other substrates for GSTT1 and genetic polymorphism in metabolism of these compounds modifies the risk of encephalopathy in human $[14,15]$.

Styrene as carcinogen that is used worldwide in the production of different polymers is also metabolized by GSTT1 enzymatic activity causing DNA and hemoglobin adducts [16]. In vivo studies are required to differentiate the genotype of GSTT1 positive and null forms to determine the enzymatic activity of GSTT1 whether it acts as conjugator(GSTT1*1)or nonconjugator enzyme (GSTT1*0).

In the present work, we analyzed the frequency of GSTT $1 * 0$ as an inactive gene product in a group of Ira- nian from Isfahan province using Real-time PCR. To get more insight into the frequency differences of defected carcinogens metaboliser gene due to deletion polymorphism of GSTT1 in this study group, results have compared with other ethnic groups.

\section{METHODS}

The participants in this study were from Isfahan province, center of Iran. Ninety healthy volunteers of both sex (41 male and 49 female). The mean age (SD; MinMax) of the individuals was 51.8 years $(51.8 ; 22-76)$. After signing the informed consent, the structured questionnaire, establishing demographic information and history of cancer in their first-degree relatives, was completed in an interview for each individual to document his or her personal and health history.

The study was approved and conducted by Institutional Review Board of Isfahan Pharmaceutical Sciences Research Center, Isfahan University of Medical Sciences, Iran.

Genomic DNA was collected from blood of each subject. DNA was extracted from lymphocytes using a Puregene DNA purification kit (Gentra Puregen, Germany). For genotyping, the thermocycler LightCycler (Roche, Mannheim, Germany) was used and hybridization probes were applied in combination with a LightCycler Fast start DNA Master Plus hybprobes kit (Roche, Mannheim, Germany). The PCR primers and probes were synthesized by TIB MOLBIOL (Berlin, Germany) according to Ko et al. method [17].

After optimization of the primers and probes concentration and temperature profiling, the final PCR conditions were optimized as follows: $0.2 \mu \mathrm{M}$ of each hybridization probe, $1 \mu \mathrm{M}$ of each primer, $2 \mu \mathrm{L}$ of the LightCycler Fast Start DNA Master Plus Hybridization Mix, and 10-50 ng of genomic DNA in a final volume of $10 \mu \mathrm{L}$. The primers and probes sequence are shown in Table 1. The temperature profiling of PCR was started with 10 min Pre-incubation time at $95^{\circ} \mathrm{C}$, Initial denaturation for

Table 1. Real-time PCR primers, Hybridization probes.

\begin{tabular}{lc}
\hline Reagents & Per sample \\
\hline $\begin{array}{l}\text { Forward Primer } \\
\text { 5'-TTCCTTACTggTCCTCACATCTC - 3' }\end{array}$ & $1 \mu \mathrm{M}$ \\
Reverse Primer & $1 \mu \mathrm{M}$ \\
5'-TCCCAgCTCACCggATCAT-3' & \\
FC probe & $0.2 \mu \mathrm{M}$ \\
5'-CCgTgggTgCTggCTgCCAAgT-FC-3' & \\
LCR640 probe & $0.2 \mu \mathrm{M}$ \\
5'-LCR640TCgAAggCCgACCCAAgCTggC-PH-3' & \\
LightCycler Fast Start DNA Master Plus Hybridization & $2 \mu \mathrm{L}$ \\
Mix & $50 \mathrm{ng}$ \\
\hline
\end{tabular}


nealing at $50^{\circ} \mathrm{C}$ and followed $20 \mathrm{Sec}$. by extension at $72^{\circ} \mathrm{C}$. Differentiation of GSTT1 genotype was performed $10 \mathrm{Sec}$. at $95^{\circ} \mathrm{C}$. Then 45 cycles amplification were performed with single acquisition followed by $15 \mathrm{Sec}$. an-by determination of melting curves after PCR. Melting curves were obtained an annealing period of $40^{\circ} \mathrm{C}$ and a final temperature of $85^{\circ} \mathrm{C}$ with a temperature gradient $0.2 / \mathrm{Sec}$ followed by cooling cycle $40^{\circ} \mathrm{C}$ for $30 \mathrm{Sec}$.

Hybridization probes (Hybprobes) consisted of two different short labeled oligonucleotides that bind to an internal sequence of the amplified fragment. The donor dye probe has a fluorescein label at the 3 -end and the acceptor dye probe has a LightCycler Red label (LightCycler Red 640) at its 5'-end.During the annealing phase, the probes are hybridized to the amplified DNA fragment, thereby bringing the two fluorescence dyes into close proximity.

Fluorescein is excited by the light source of the Light Cycler system, which causes to emit green fluorescence light. The emitted energy excites LightCycler red fluorescence resonance energy transfer (FRET).The red fluorescence is measured at the end of each annealing step, when the fluorescence intensity is greatest [18].

Intra-assay (the amount of the error seen with the same assay in each run) and inter-assay (the error between separate assays) were performed.

Statistical significance of differences between age, gender and GSTT1 genotypes was calculated by the chi-squared or Fisher exact tests. The chi-squared test was performed to investigate differences between the frequencies of the null genotypes in the specified areas . The probability $\mathrm{P}<0.05$ was considered statistically significant. All the P-values were two-tailed. Analyses were performed using the statistical package SPSS for windows, version 12 (SAS Institute, NC, USA)

\section{RESULTS}

Sufficient quantities of DNA were obtained from all specimens. A total of 90 individuals were genotyped for GSTT1 by use of Real-time PCR. Table 2 shows the percentage of GSTT1 variants by age and gender.

The frequency of GSTT1*1 was $77.4 \%$ in the individuals older than 60 years and $72.8 \%$ in the younger ones (age less than 60 years). GSTT $1 * 0$ was $27.1 \%$ in the group of below 60 years versus $22.6 \%$ in subjects above 60 years. No differences in genotype frequency were perceived in samples stratified by age ( $p$-value $=$ 0.8 ). In Table 2 also frequency by gender is shown. Totally $73.5 \%$ of females showed GSTT $1 * 1$ genotype and $26.5 \%$ null genotype. The frequency of GSTT1 null genotype in male group was $24.4 \%$ and GSTT1*1was $75.6 \%$. No significant difference between gender was seen with GSTT1 genotype (p-value $=1)$.
Table 2. Frequency of GSTT1 genotypes by age and gender.

\begin{tabular}{llll}
\hline Features & Number $(\%)$ & GSTT1*1 & GSTT1*0 \\
\hline & & N $(\%)$ & N $(\%)$ \\
Age & & & \\
$\leq 60$ & $59(65.5 \%)$ & $43 / 59(72.8 \%)$ & $16 / 59(27.2 \%)$ \\
$>60$ & $31(34.4 \%)$ & $24 / 31(77.4 \%)$ & $7 / 31(22.6 \%)$ \\
p-value & & 0.8 & \\
Gender & & & $13 / 49(26.5 \%)$ \\
Female & $49(54.4)$ & $36 / 49(73.5 \%)$ & $10 / 41(24.4 \%)$ \\
Male & $41(45.6)$ & $31 / 41(75.6)$ & \\
p-value & & 1 & \\
\hline
\end{tabular}

The differences of GSTT $1 * 0$ genotype frequency were marginally significant in comparision with some East Asian countries (China $\chi^{2}=19.16, \mathrm{df}=1, \mathrm{P}<0.05$, Shanghai $\chi^{2}=14.22, \mathrm{df}=1, \mathrm{P}<0.05$, Korea $\chi^{2}=18.40$, $\mathrm{df}=1, \mathrm{P}<0.05$, Japan $\chi^{2}=18.53, \mathrm{df}=1, \mathrm{P}<0.05$ ).

The result of our study are shown significant differences with Caucasian $\chi^{2}=5.23, \mathrm{P}<0.05$ and Mexican-Americans $\chi^{2}=5.6 .84, \mathrm{P}<0.05$ ).

\section{DISCUSSION}

Since the occurrence of the GSTT1 polymorphism seems to be strongly dependent on ethnic origin, we investigated the frequency of the GSTT1 genotypes in our study group for comparing with the other groups as well as with other countries population. In our study the prevalence of GSTT $1 * 0$ was $25.5 \%$. This result was slightly higher than Turkish, Mexican-Americans and Caucasians but lower than Chinese, Korean and Japanese (Table 3). The reported frequency for other Iranian groups also presented significant differences between Iranian populations [19]. Iran is a big country with an estimated 70.5 million people that they are ethnically diverse. At least 8 different ethnic groups live in Iran. Our study group selected from Persian Muslims who living in Isfahan province. Isfahan is third largest city in Iran with more than 1.500 .000 populations. It should be considered that many people have immigrated from other cities with different ethnicity to Isfahan specially from the Iranian-Iraqi border province (Khuzestan) in southwest of Iran since Iran-Iraq war (1981-1989). They had different ethnicity "Iranian Arab" and this people have still stayed there. It means the study group could be heterogeneous thus may be different from other Persian population in Iran. It should be noted despite of different frequencies in present study from other Iranian ethnic groups which was reported by Rafiee in 2010 [19] there 
were no statistically significant differences (Iranian Georgian, $\chi^{2}=1.66, d f=1, p=0.197$ and Iranian Persian, $\chi^{2}$ $=3.34, d f=1, \mathrm{p}=0.068$ ).

According to result of worldwide studies in different ethnic groups (Table 3) it is completely obvious that there is a wide range differences between populations. The frequency of the null genotype is highest among Chinese (64.4\%) followed by Koreans and Japanese [20]. GSTT $1 * 0$ is much more frequent $(64 \%-54 \%)$ in this area populations [21] comparing with the others. Germans [17] and Caucasians [22] and New England [23] have shown less frequency (12-18\%) and the prevalence was low among Mexican-American [24]. However, some gradient and intra ethnic differences are reported as well, such as Shanghai [21], Turkey [21], Korea [25-27] and Iran [19]. The chi-squared and p-values have been shown in Table 3. GSTT $1 * 0$ genotype showed a distribution profile regarding to geographical area. It is more likely the pattern of deletion polymorphism of GSTT1 shows a geographical distribution from East Asian countries to middle Asia followed by European countries to Americans.
According to our result in comparison with other studies, Iranians showed the middle frequency for this null genotype. As it has shown in Table 3 our study group significantly $(\mathrm{P}<0.05)$ differs from East Asian countries and Caucasians, Mexican Americans and Turkish.

Following the identification of the deletion in GSTT1 gene deletion underlying the impaired capacity for detoxification of environmental pollutant and carcinogens, it was reported that a wide range differences present in different population.

The prospect of screening in the gene level for prevention, prediction or early diagnosis of some diseases especially cancer is becoming real. A vast number of studies are focused on this aim, to investigate whether genetic variation between populations contributes to different capacity for detoxifying carcinogens and consequently susceptibility to cancers as well as relapse [28,29].

Moving toward individualized drug treatment as a new and very interesting approach attracts so many attention to defining the polymorphic genes coding for drug metabolizing enzyme and prediction of anticancer drug [30-32].

Table 3. Frequency GSTT1 genotypes in the present study, in comparison to those in other studies in worldwide populations.

\begin{tabular}{|c|c|c|c|c|c|c|c|}
\hline Group & Population & Reference & Number & \%GSTT1*0 & $\%$ GSTT1*1 & Chi square $d f=1$ & $P$ value \\
\hline \multicolumn{8}{|c|}{ East Asian } \\
\hline 1 & Chinese & 36 & 45 & 64.4 & 35.6 & 19.16 & $\mathrm{P}<0.05$ \\
\hline 2 & Shanghai & 14 & 219 & 49 & 51 & 14.22 & $\mathrm{P}<0.05$ \\
\hline 3 & Koreans1 & 28 & 177 & 53.1 & 46.9 & 18.40 & $\mathrm{P}<0.05$ \\
\hline 4 & Koreans2 & 30 & 1037 & 54.3 & 45.7 & 27.39 & $\mathrm{P}<0.05$ \\
\hline 5 & Koreans3 & 29 & 273 & 55.1 & 44.9 & 23.43 & $\mathbf{P}<\mathbf{0 . 0 5}$ \\
\hline 6 & Japanese & 26 & 150 & 54 & 46 & 18.53 & $\mathbf{P}<0.05$ \\
\hline \multicolumn{8}{|c|}{$\begin{array}{l}\text { European and } \\
\text { Americans }\end{array}$} \\
\hline 7 & Caucasians & 31 & 213 & 14.7 & 85.3 & 5.22 & $\mathrm{P}<0.05$ \\
\hline 8 & Germans & 17 & 90 & 17 & 83 & 2.13 & $P=0.144$ \\
\hline 9 & New England & 32 & 185 & 15.7 & 84.3 & 3.85 & $\mathbf{P}=\mathbf{0 . 0 5}$ \\
\hline 10 & Mexican-Americans & 27 & 73 & 10 & 90 & 6.84 & $\mathrm{P}<\mathbf{0 . 0 5}$ \\
\hline 11 & Brazilians & 34,35 & 594 & 23.1 & 76.9 & 0.31 & $\mathbf{P}=\mathbf{0 . 5 7}$ \\
\hline \multicolumn{8}{|c|}{ Middle East Asian } \\
\hline 12 & Turkish & 33 & 128 & 7 & 93 & 14.48 & $\mathrm{P}<0.05$ \\
\hline 13 & IraniansGeorgian & 25 & 34 & 15.7 & 84.3 & 1.66 & $\mathbf{P}=\mathbf{0 . 1 9 7}$ \\
\hline 14 & Iranians Persian & 25 & 249 & 35.5 & 64.5 & 3.34 & $P=0.068$ \\
\hline 15 & Iranians Shiraz & 25 & 121 & 24.8 & 75.2 & 0.016 & $\mathbf{P}=\mathbf{0 . 9 0}$ \\
\hline 16 & Iranians & Present study & 90 & 25.5 & 74.5 & & \\
\hline
\end{tabular}


GSTT1* 0 genotype is one of the well known polymorphism of this enzyme which represent a defect phenotype to metabolize and biotransformation process. The relationship between GSTs genes polymorphism and diseases has been investigated in so many publications since 1990. Regarding to the role of GSTs in detoxification of carcinogens the common question was: Is there any association between different polymorphism in GSTs gene polymorphism and risk of specific cancers?

A PubMed search using the "GSTT1 cancer risk" retrieved 767 references, "GSTT1 null genotype cancer risk" retrieved 478 Articles (January 2011). Some of the HuGE review articles and Meta analysis specified in GST gene polymorphism and cancer risk.

Raimondi and colleagues (2006) performed a Meta and pooled analysis of GSTT1 and lung cancer they failed to show association between GSTT1 null genotype and lung cancer in Caucasians but in Asian the association has been showed [33]. Liao et al (2010) achieved that there was a small increased risk of colorectal cancer for individuals with GSTT1 null, especially for Caucasians populations and Asian populations [34]. In 2010 Wan and colleague demonstrated that GSTT1 null genotype is associated with an increased risk of colorectal cancer, specifically, among Caucasians [35]. The GSTT1 null genotype was found to be associated with increased risks of tumors of the stomach [36,37], bladder $[38,39]$. The results of Ha YS et al study at 2010 suggested that the GSTT1 genotype could be a useful prognostic marker for recurrence and progression in nonmuscle-invasive bladder cancer [40].

Hence $\mathrm{GSTT} 1{ }^{*} 0$, is a potential genetic risk marker for different diseases it seems necessary to use a rapid and accurate technique for analyzing the GSTT1 genotypes. The Real-time PCR that we used in this study which is followed by fluorescence monitoring of dissociation hybridization probes from DNA amplicon, provide a sensitive, quick, safe and one step method for using in clinical screening.

More of the studies for genotyping the GSTT1 gene used conventional PCR and gel electrophoresis based documentation methods but in this study the LightCycler system was used. Unlike conventional PCR, in which cycling takes several hours, PCR analysis conducted with the LightCycler took around 1 hour. In contrast to previous assays that used in majority of studies which depended on the analysis of Ethidium bromide-stained gels (with consideration of toxicity), no additional time consuming procedure (e.g., casting agarose gel, staining, or southern blotting analyses) were required to analyze the PCR product by use of LightCycler. In this study the amplified products were identified by the online melting peak analysis, allowing more reliable distinction be- tween genotypes. Direct, uncomplicated analysis of the results also reduced the number of handling steps, thus minimizing the risk of sample contamination.

Although we used a set of HybProbe that is relatively expensive but rapid and one-step method provide a time-effective also cost-effective method for genotyping a large number of samples without any handling of Ethidium bromide as a toxic material. It possible to analyze and document several samples simultaneously in each runs of LightCycler around one hour.

The frequency of GSTT $1 * 0$ in the group of Iran is different from some Asian countries. In base of wide spectrum of frequency differences for GSTT1 genotypes that have been already documented, it seems to be necessary to use a rapid and reliable method for GSTT1 genotyping in each single toxicology, epidemiology and clinical case-control studies. This study can be expanded by: increasing the sample size, investigation of modifiers like age and gender, designing the case-control studies and similar studies in other functional genes mutation.

\section{ACKNOWLEDGEMENT}

Special thanks to management of Day General Hospital Laboratory and its Molecular Diagnosis Department, especially Dr. Farzaneh Rahimi and Mr. Ramin Naghizadeh for their invaluable support. Authors are also thankful of Professor Mohammad Abdollahi from Faculty of Pharmacy in Tehran University of Medical Sciences for editing the manuscript. We also thank TIB MOLBIOL, Berlin, for the construction of the primers and LightCycler Hybridization Probes and technical suggestions. This work was supported by the Research Council of the Isfahan University of Medical Sciences, Isfahan, Iran.

\section{REFERENCES}

[1] Mcillwain, C.C., Townsed, D.M. and Tew, K.D. (2006) Glutathione S-transferase polymorphisms: cancer incidence and therapy. Oncogene, 25, 1639-1648. doi:10.1038/sj.onc.1209373

[2] Strange, R.C., Jones, P.W. and Fryer, A.A. (2000) Glutathione S-transferase genetics and role in toxicity. Toxicology Letters, 112, 357-363. doi:10.1016/S0378-4274(99)00230-1

[3] Wen, H. and Ali-osman, F. (2007) Genetic polymorphism and function of glutathione S-transferase in tumor drug resistance. Current Opinion in Pharmacology, 7, 367-374. doi:10.1016/j.coph.2007.06.009

[4] Jang, S.G., Kim, I.J. et al. (2007) GSTT2 promoter polymorphisms and colorectal cancer risk. BMC Cancer, 25, 7-16.

[5] Shewita, S.A. and Timisany, A.K. (2003) Cancer and Phase II drug-metabolizing enzymes. Current Drug Metabolism, 4, 45-58. doi:10.2174/1389200033336919

[6] Sivonova, M., Wakzuliva, I. et al. (2009) Polymorphisms of glutathione S-transferase M1, T1, P1 and the risk of prostate cancer. Journal of Experimental \& Clinical Cancer Research, 5, 28-32.

[7] Zarte, R.R., Morales, R. et al. (2006) Potential applica- 
tion of GSTT1-null genotype in predicting toxicity associated to 5-fluouracil irinotecan and leucovorin regimen in advanced stage colorectal cancer patients. Oncology Reports, 16, 497-503.

[8] Giovanni, M. and Marco, M. (2007) Risk assessment of occupational exposure to polycyclic aromatic hydrocarbons by means of urinary1-hydroxypyrene. Toxicology and Industrial Health, 23, 55-59.

[9] Goodman, J.E. and Mechanic, L.E. (2006) Exploring "SNP-SNP interactions and colon cancer risk using polymorphism interaction analysis". International Journal of Cancer, 118, 1790-1797.

[10] Norppa, H. (2004) Cytogenetics biomarkers. IARC Scientific Publications, 157, 179-205.

[11] Olvera-Bello, A.E. et al. (2010) Susceptibility to the cytogenetic effects of dichloromethane is related to the glutathione S-transferase theta phenotype. Toxicology Letters, 199, 218-224. doi:10.1016/j.toxlet.2010.09.002

[12] National Toxicology Program (2010) Final Report on Carcinogens Background Document for Formaldehyde. Final Report on Carcinogens Background Document, 10, i-512.

[13] Casanova, M., Bell, D.A. and Heck, H.A. (1997) Dichloromethane metabolism to formaldehyde and reaction with nucleic acids in hepatocytes of rodents and humans with and without glutathione S-transferase T1 and M1 genes. Fundamental and Applied Toxicology, 37, 168-180. doi:10.1006/faat.1997.2313

[14] Landi, S. (2000) Mammalian class theta GST and differential susceptibility to carcinogenesis: a review. Mutation Research, 463, 247-283. doi:10.1016/S1383-5742(00)00050-8

[15] Kezic, S. and Calkoen, F. (2006) Genetic polymorphism of metabolic enzymes modifies the risk of chronic solvent-induced encephalopathy. Toxicology and Industrial Health, 22, 281-289. doi:10.1177/0748233706070287

[16] National Toxicology Program (2008) Final Report on Carcinogens Background Document for Styrene. Final Report on Carcinogens Background Document, 8, i-398.

[17] Ko, K., Koch, B. et al. (2000) Rapid analysis of GSTM1, GSTT1, GSTP1 polymorphisms using real-time polymerase chain reaction. Pharmacogenetics, 10, 1-4. doi:10.1097/00008571-200004000-00009

[18] Light Cycler Fast Start DNA Master PlusHybProbe. www.roch-applied-science.com

[19] Huang, M. and Wang, Y. (2007) Multiple genetic polymorphism of GSTP1 313AG, MDR1 3435CC, and MTHFR 677CC highly correlated with early relapse of breast cancer patients in Taiwan. Annals in Surgical Oncology, 15, 872-880. doi:10.1245/s10434-007-9719-7

[20] Deeken, J.F., Figg, W.D. et al. (2007) Toward individualized treatment: prediction of anticancer drug disposition and toxicity with pharmacogenetics. Anticancer Drugs, 18, 111-126. doi:10.1097/CAD.0b013e3280109411

[21] Bosch, T.M. (2008) Pharmacogenomics of drug-metabolizing enzymes and drug transporters in chemotherapy. Methods in Molecular Biology, 448, 63-76.

[22] Efferth, T. and Volm, M. (2005) Pharmacogenetic for individualized cancer chemotherapy. Pharmacology \& Therapeutics, 107, 155-76.
[23] Ahmadi, A. and Jönsson, P. (2002) Interaction between smoking and glutathione S-transferase polymorphisms in solvent-induced chronic toxic encephalopathy. Toxicology and Industrial Health, 18, 289-296.

[24] Parl, F.F. (2005) Glutathione S-transferase genotypes and cancer risk. Cancer Letters, 221, 123-129. doi:10.1016/i.canlet.2004.06.016

[25] Rafiee, L., Saadat, I. and Saadat, M. (2010) Glutathione S-transferase genetic polymorphisms (GSTM1, GTT1 and GSTO2 in three Iranian populations. Molecular Biology Reports, 37, 155-158. doi:10.1007/s11033-009-9565-8

[26] Nelson, H.H., Wiencke, J.K. et al. (1995) Ethnic differences in the prevalence of the homozygous deleted genotype of glutathione S-transferase theta. Carcinogenesis, 16, 1243-1245. doi:10.1093/carcin/16.5.1243

[27] Naoe, T. and Takeyama, K. (2000) Analysis of the genetic polymorphism in NQ01, GSTM1, GSTT1, and CYP3A4 in 469 Japanese patients with therapy-related leukemia, meylodysplastic syndrome and the acute myeloid leukemia. Clinical Cancer Research, 6, 4091-4095.

[28] Cho, H., Lee, S. et al. (2005) GSTM1, GSTT1 and GSTP1 Polymorphism in the Korean population. Journal of Korean Medical Science, 20, 1089-1092. doi:10.3346/jkms.2005.20.6.1089

[29] Jang, S.S., Jung, C.Y. et al. (2003) The GSTT1 genotype as a marker for susceptibility to lung cancer in Korean female never smoker. Tuberculosis and Respiratory Diseases, 54, 485-494.

[30] Choe, S.C., Yun, K.J. et al. (2003) Prognostic potential of glutathione S-transferase M1 and T1 null genotypes for gastric cancer progression. Cancer Letters, 195, 169-175.

[31] Chen, C.L., Liu, Q. and Relling, M.W. (1996) Simultaneous characterization of glutathione S-transferase M1 and $\mathrm{T} 1$ polymorphisms by polymerase chain reaction in American whites and blacks. Pharmacogenetics, 6, 187-191. doi:10.1097/00008571-199604000-00005

[32] Nelson, H.H., Wiencke, J.K. et al. (1995) Ethnic differences in the prevalence of the homozygous deleted genotype of glutathione S-transferase theta. Carcinogenesis, 16, 1243-1245. doi:10.1093/carcin/16.5.1243

[33] Raimondi, S., Paracchini, V. et al. (2006) Meta- and pooled analysis of GSTT1 and lung cancer: a HuGE-GSEC review. American Journal of Epidemiology, 164, 1027-1042. doi:10.1093/aje/kwj321

[34] Liao, C., Cao, Y.F. et al. (2010) An updating metaanalysis of the glutathione S-transferaseT1 polymorphisms and colorectal cancer risk: a HuGE review. International Journal of Colorectal Disease, 25, 25-37. doi:10.1007/s00384-009-0805-0

[35] Wan, H.W., Zhou, Y. et al. (2010) Genetic polymorphism of glutathione S-transferase T1 and the risk of colorectal cancer: a meta-analysis. Cancer Epidemiology, 34, 66-72.

[36] Boccia, S., La, T.G. et al. (2006) Glutathione stransferase T1 status and gastric cancer risk: a meta-analysis of the literature. Mutagenesis, 21, 115-123. doi:10.1093/mutage/gel005

[37] Saadat, M. (2006) Genetic polymorphisms of glutathione S-transferase T1 (GSTT1) and Susceptibility to gastric cancer: a meta-analysis. Cancer Science, 97, 505-509. doi:10.1111/j.1349-7006.2006.00207.x

[38] Abdel-Rahman, S.Z., Anwar, W.A. et al. (1998) GSTM1 
and GSTT1 genes are potential risk modifiers for bladder cancer. Cancer Detection and Prevention, 22, 129-138. doi:10.1046/j.1525-1500.1998.00934.x

[39] Grando, J.P. and Kuasne, H. (2009) Association between polymorphisms in the biometabolism genes CYP1A1, GSTM1, GSTT1 and GSTP1 in bladder cancer. Clinical and Experimental Medicine, 9, 21-28. doi:10.1007/s10238-008-0015-Z

[40] Ha, Y.S., Yan, C. et al. (2010) GSTT1 as a prognosticator for recurrence and progression in patients with non-muscle invasive bladder cancer. Disease Markers, 29, 81-87. 